\title{
A Small Spot, Inert Gas, Ion Milling Process as a Complementary Technique to Focused Ion Beam Specimen Preparation
}

\author{
Paul E. Fischione, ${ }^{1, *}$ Robert E.A. Williams, ${ }^{2}$ Arda Genç, ${ }^{2}$ Hamish L. Fraser, ${ }^{2}$ \\ Rafal E. Dunin-Borkowski, ${ }^{3}$ Martina Luysberg, ${ }^{3}$ Cecile S. Bonifacio, ${ }^{1}$ and András Kovács ${ }^{3}$ \\ ${ }^{1}$ E.A. Fischione Instruments Inc., 9003 Corporate Circle, Export, PA 15632, USA \\ ${ }^{2}$ Center for the Accelerated Maturation of Materials, The Ohio State University, 1305 Kinnear Road, Columbus, OH 43212, USA \\ ${ }^{3}$ Ernst Ruska-Centre for Microscopy and Spectroscopy with Electrons and Peter Grünberg Institute, Forschungszentrum Jülich \\ GmbH, Wilhelm-Johnen-Straße, 52425 Jülich, Germany
}

\begin{abstract}
This paper reports on the substantial improvement of specimen quality by use of a low voltage $(0.05$ to $\sim 1 \mathrm{keV})$, small diameter $(\sim 1 \mu \mathrm{m})$, argon ion beam following initial preparation using conventional broad-beam ion milling or focused ion beam. The specimens show significant reductions in the amorphous layer thickness and implanted artifacts. The targeted ion milling controls the specimen thickness according to the needs of advanced aberration-corrected and/or analytical transmission electron microscopy applications.
\end{abstract}

Key words: ion milling, focused ion beam, amorphous damage, implantation, artifact

\section{INTRODUCTION}

Significant information about a material's chemistry, magnetic properties, atom locations, surface characteristics, and microstructure can be obtained through transmission electron microscopy (TEM), which requires artifact-free specimens to quantify these properties in two and three dimensions.

The TEM specimen preparation techniques used most commonly involve broad-beam Ar ions or focused ion beam (FIB), milling using Ga ions in a scanning electron microscope. Surface damage and unintended ion-implanted layers incurred during the preparation process are some artifacts that limit the information that is obtainable from analytical and high-resolution electron microscopy. Deleterious surface layers are often a significant fraction of the total specimen thickness, which affects both the quality and quantity of the results.

Within the past 20 years, FIB systems incorporating Ga ion beams of up to $50 \mathrm{keV}$ have been applied to preparing specimens for TEM analysis from almost any solid material. The thinning and extraction of TEM specimens with accuracy and site-specificity is an advantage of FIB (Kirk et al., 1989; Young et al., 1990; Basile et al., 1991; Giannuzzi \& Stevie, 1999; Anderson \& Klepeis, 2005; Kamino et al., 2005; Jia et al., 2014). A significant fraction of the specimens produced at any given laboratory is now prepared using FIB technology.

Advances in aberration-corrected TEM imaging, monochromation, and enhanced analytical methods have been achieved during the past decade (Batson et al., 2002).

Received October 3, 2016; accepted April 6, 2017

*Corresponding author. pe_fischione@fischione.com
Techniques such as energy-dispersive $\mathrm{X}$-ray spectroscopy (EDXS), electron energy-loss spectroscopy (EELS), high-angle annular dark-field (HAADF) detection, and energy-filtered imaging are routinely used for elemental identification and quantification. Electron tomography for three-dimensional imaging has also become a common technique for the physical sciences.

These improvements in the resolution and analytical capabilities of modern microscopes require the minimization of artifacts incurred during the preparation process. For example, artifacts of specimen thinning with a Ga ion beam may include surface amorphization, Ga implantation, and the generation of crystallographic defects (Barna, 1991; Barber, 1993; Barna et al., 1999; McCaffrey et al., 2001; Kato, 2004; Anderson \& Klepeis, 2005; Mayer et al., 2007; MoberlyChan et al., 2007; Volkert \& Minor, 2007). Similarly, specimens prepared by electrolytic polishing or conventional mechanical grinding followed by broad-beam ion-thinning methods can also exhibit surface damage (Barna, 1991; Barna et al., 1999). Disordered surface layers, such as thin native oxides, have also been shown to contribute significantly to surface plasmon excitations in low-loss EELS (Scheu et al., 2003; Mkhoyan et al., 2007).

Low energy $(<1 \mathrm{keV})$, inert gas ion milling is an attractive method (Barna, 1991; Barber, 1993; Barna et al., 1999; Kato, 2004; Genç et al., 2007; Mayer et al., 2007; MoberlyChan et al., 2007; Volkert \& Minor, 2007; Miyajima et al., 2010; Mehrtens et al., 2012; Lotnyk et al., 2015) for lessening the effects of surface damage. For crystalline materials, the ideal procedure is to reduce progressively the specimen thickness by removing surface damage layers while leaving the material beneath undisturbed. Surface damage can constitute a substantial 
fraction of the total specimen thickness (Giannuzzi, 2006); therefore, reducing the layer thickness can yield thin specimens that are ideal for a variety of imaging and analytical techniques.

For example, the use of high-resolution transmission electron microscopy (HRTEM) and EELS often require specimens to be thinned to meet weak-phase object criteria and ideally represent the single scattering regime. The preparation challenge is compounded for small $(\sim 10 \times 5 \mu \mathrm{m})$ FIB lift-out specimens because post-FIB damage removal based upon broad-beam ion milling risks redeposition, that is, a broad beam covers not only the area of interest, but also affects other parts of the specimen, such as the support grid onto which the FIB lamella is mounted and the protective cap layer. As a consequence, material from the grid and the cap are sputtered and partly redeposited onto the specimen. Therefore, the ability to focus and selectively target the milling area is highly desirable to avoid unintended sputtering and redeposition.

One of the most important criteria for TEM specimen preparation is to produce an electron-transparent region that is representative of the bulk material's structure and properties while reducing preparation-induced artifacts to a minimum. The primary preparation-induced artifacts generated during ion milling are the following:

Ion implantation: The accelerated Ga ions penetrate the specimen surface creating an artificial layer that has different properties than the bulk material. Such "dead layer" formation is a major problem for studies of semiconductor properties in $p-n$ junctions or magnetic materials (Cooper et al., 2009). Post-FIB, low energy, Ar ion milling is able to remove the Ga-contaminated layer because $\mathrm{Ar}$ is lighter than Ga and rarely forms chemical bonds with other elements (Unocic et al., 2010).

Amorphization: For both Ga and Ar ions, the high-energy ionic particles break the chemical bonds of the crystalline materials and create an amorphous layer on the surface. Formation of amorphous layer is materials-dependent; semiconductor and ceramic materials easily amorphize when compared with metals (Huh et al., 2013). Decreasing the energy and the incident angle of the incoming ions can reduce the effect (Barna et al., 1998).

Surface roughness: The sputtering ions create volume defects due to surface inhomogeneities and the resulting sputtering rate differences. Typically, ceramics, oxides, and multi-phase alloy systems are sensitive to milling-induced surface roughness. The degree of surface roughness is exacerbated when specimens contain layers or phases with different sputtering rates, for example, substrate (sputtering rate $\left.V_{1}\right)$, layer $\left(V_{2}\right)$, protective layer $\left(V_{3}\right)$, and $V_{1} \neq V_{2} \neq V_{3}$. Low angle, low energy, Ar ion milling, combined with either beam rastering or specimen oscillation, can reduce the effect.

Redeposition: $\mathrm{Cu}, \mathrm{Pt}, \mathrm{Au}$, or other sputtered material from the FIB lamellae support and the protective cap layer can redeposit onto the surface of lamellae. The effect can be fully eliminated by focusing the Ar ion beam at the lamellae and by positioning the lamellae on the support grid in a way that ions only interact with the area of interest on the lamellae. This method is made possible by the use of Everhart-Thornley detector technology to image ion-induced secondary electrons, which provides an exact representation of the area being milled.

Heating: Sputtering of the surface is a localized thermal effect associated with the energy applied during milling, which can lead to amorphization, intermixing, and selective etching of the materials. Cooling the specimen during ion milling can reduce the effect (Bahnck \& Hull, 1990).

The benefits of low energy, inert gas (Ar) ion milling for the removal of amorphous and implanted surface layers, and the improvement of microscopy results are described in this paper.

\section{FIB Lift-Out and Focused Ar Ion Beam Milling}

TEM specimen preparations of semiconductor materials systems will be presented that represent characteristic cases for surface amorphization and roughness. An example for beam-sensitive material that required low-voltage TEM and very thin specimen thickness is also presented.

For conventional ion beam technology, the size of the $\mathrm{Ar}$ ion beam can vary from $\sim 300 \mu \mathrm{m}$ to $1.5 \mathrm{~mm}$ full-width at half-maximum (FWHM). The advantage of broad-beam technology is that ions can be generated at a sufficiently low energy to minimize specimen damage; however, at low energy, the ion beam diameter can greatly increase to a few millimeters. In a FIB, the Ga ion beam diameter can be on the order of $5 \mathrm{~nm}$ (Utke et al., 2008). An advantage of FIB is that the use of a liquid metal $(\mathrm{Ga})$ nanometer-sized beam is often combined with an electron column for imaging during the preparation process, which enables site-specific specimen preparation. However, specimen thinning with a $\mathrm{Ga}$ ion beam at sufficiently low energy can be a time-consuming process and requires a skilled user and a very stable, well-aligned system (Schaffer et al., 2012).

The challenge was to develop a technology that used inert ions to avoid chemical alteration, low energy to minimize amorphous layers, low temperature to reduce heat-induced damage, and a small ion spot to prevent redeposition. With these criteria, a unique ion source technology was developed: an ion beam of sufficiently low energy (as low as $50 \mathrm{eV}$ ), concentrated into a small spot (as small as $1 \mu \mathrm{m}$ ), that produced sufficient current $(\sim 100-150 \mathrm{pA})$ to allow for reasonable milling rates $(\sim 1-8 \mathrm{~nm} / \mathrm{min})$. Milling rates are dependent upon the accelerating voltage of the incident ions, the corresponding beam current, the milling angle, and the size of the area targeted by the rastered ion beam. To investigate the effect of a small-spot Ar ion beams to produce highquality electron transparent specimens, TEM and scanning transmission electron microscopy (STEM) specimens of several important materials classes were chosen for this study.

The ion source developed for this application uses electrons from a thermionic emitter (filament) to ionize argon gas. A series of electrodes, biased at voltages relative to the filament, guide the primary electrons into an ionizing chamber. A mass flow controller regulates argon gas flow. Collisions between 
electrons and gas atoms produce ions that are primarily positive and singularly charged. Some of the ions escape through an aperture and are accelerated through an electrostatic lens. The result is that ions impact only a small area of the specimen. Varying the bias on the lens changes its focal plane and allows the spot size to be adjusted. The voltage of the ionizing chamber determines the final particle energy at the target (the specimen), which is maintained at ground potential. Electrostatic deflectors, located at the exit of the lens, steer the beam transverse from its main axis in two directions (horizontal and vertical), which allows positioning of the spot relative to the target. Under computer control, the deflectors can scan the beam across a field of view or locate it at arbitrary points.

To establish the position of the ion beam with respect to the specimen, an Everhart-Thornley-type secondary electron detector (SED) was incorporated to image ion-induced secondary electrons. The ion source was fitted to a vacuum chamber possessing a specimen stage that can both rotate the specimen to achieve an appropriate initial orientation and tilt to yield milling angle adjustability. To further reduce the possibility of specimen damage, the stage was thermally attached to a liquid nitrogen dewar that allows ion milling to occur at cryogenic temperatures (approximately $-170{ }^{\circ} \mathrm{C}$ ). The chamber was also equipped with a Faraday cup to monitor ion beam current.

\section{Materials and Methods}

The materials investigated in this study include semiconductor $\mathrm{Si}$ (Figs. 1, 2); MnAs on GaAs (Figs. 3, 4); oxide semiconductor Co: $\mathrm{ZnO}$ on $\mathrm{Al}_{2} \mathrm{O}_{3}$ (Fig. 5); a multilayer of $\mathrm{CaTiO}_{3}$ and $\mathrm{SrTiO}_{3}$ (Fig. 6); a $\mathrm{Sr}_{2} \mathrm{FeMoO}_{6}$ thin film grown on $\mathrm{SrTiO}_{3}$ (Fig. 7); a Ni-based superalloy (Fig. 8); and a structural Ti alloy (Figs. 9, 10) (Li \& Yang, 2002). The application examples of the benefit of low-energy-focused ion milling presented required different specimen thicknesses in the range of few nanometers to a few tenths of nanometers according to the imaging or spectroscopy characterization methods.

Specimens of Si milled with a Ga ion beam were first prepared to assess the surface chemistry and thickness of damage. To best prepare FIB lift-out specimens for TEMEDXS analysis, a capping layer of $1-2 \mu \mathrm{m}$ of Pt was electron beam deposited to protect the as-milled surfaces from further alteration by the Ga ion beam. HRTEM and STEM imaging and microanalysis were conducted. Specimens for the other TEM analyses in this work were prepared by mechanical polishing and conventional broad-beam Ar ion milling.

The FIB specimens were prepared using a Helios NanoLab 400S dual beam system (Thermo Fisher Scientific, Waltham, MA, USA) equipped with a micromanipulator (Omniprobe, Oxford Instruments NanoAnalysis, High Wycombe, UK) and gas injection system for deposition of $\mathrm{Pt} / \mathrm{C}$ and pure $\mathrm{C}$ protective layer on the surface of the specimen. Post-FIB, low energy $(<1 \mathrm{keV})$ Ar ion milling was conducted using $\sim 1-\mu \mathrm{m}$ diameter concentrated ion beam in a NanoMill TEM specimen preparation system (E.A. Fischione Instruments, Inc., Export, PA, USA). The steps of the post-FIB ion milling processes and the important parameters are listed in Table 1.
Both conventional and aberration-corrected TEM observations were used to image the ion-milled specimens. In some cases, conventional TEM was preferred as a quality check of the specimens because it is more sensitive to surface inhomogeneities caused by image contrast than the aberration-corrected TEM images that usually do not use an objective aperture for image recording. For TEM studies, the following microscopes were used: a TEAM 0.5 (United States Department of Energy, Lawrence Berkeley National Laboratory, Berkeley, CA, USA) operated at $80 \mathrm{keV}$, Tecnai G2 and TF20 (Thermo Fisher Scientific) operated at $200 \mathrm{keV}$, a CM200 (Philips, Koninklijke Philips N.V. Amsterdam, The Netherlands), and an aberration-corrected Titan (Thermo Fisher Scientific) operated at 80 and $300 \mathrm{keV}$.

The decrease in surface damage was characterized between milling sessions by applying fast Fourier transforms (FFT) on the acquired images, which indicate the periodicities conveyed by the objective lens in phase contrast imaging. The diffuse halo contrast, located near the central region of the FFT, is associated empirically with surface amorphization, such that the number of frequencies present in the FFT increase and the central halo decreases as the amorphous surface layer is reduced. By initially considering a material's sputtering threshold and progressively reducing the Ar ion beam energy, a steady-state operating point is desiredwhereby the rate of damage removal outweighs the potential for the creation of new damage (Barnard et al., 2006).

Different dual beam systems may have different parameters and additional steps in TEM specimen preparation. Table 1 summarizes the main procedure and parameters of TEM specimen preparation using FIB and NanoMill system that became a standard procedure at the Ernst Ruska-Centre. Because the NanoMilling process is incorporated into the specimen preparation procedure, low-energy $\mathrm{Ga}$ ion milling $(<5 \mathrm{keV})$ in the FIB is omitted.

The thickness of the electron beam-deposited protective layer is typically in a range of $100-500 \mathrm{~nm}$, whereas the ion beam deposited Pt/C layer is $\sim 3-\mu \mathrm{m}$ thick. Deposition of amorphous carbon and/or the $\mathrm{Pt} / \mathrm{C}$ layers on the surface of the lamellae can be advantageous in tuning the aberration corrector of the microscope.

\section{Results And Discussion}

\section{Beam Size and Surface}

TEM images collected before and after targeted, low-energy Ar ion milling ${ }^{a}$ demonstrate that the amorphous damage layer has been reduced in thickness, as will be demonstrated in the following.

The removal of thin, damaged surface layers necessitates the use of relatively slow milling rates to avoid over-thinning the lamella, which could result in diminished structural integrity of the specimen. Atomic force microscopy was used

\footnotetext{
a Throughout the paper "Ar ion milling" refers to small spot Ar ion milling, if not stated otherwise.
} 

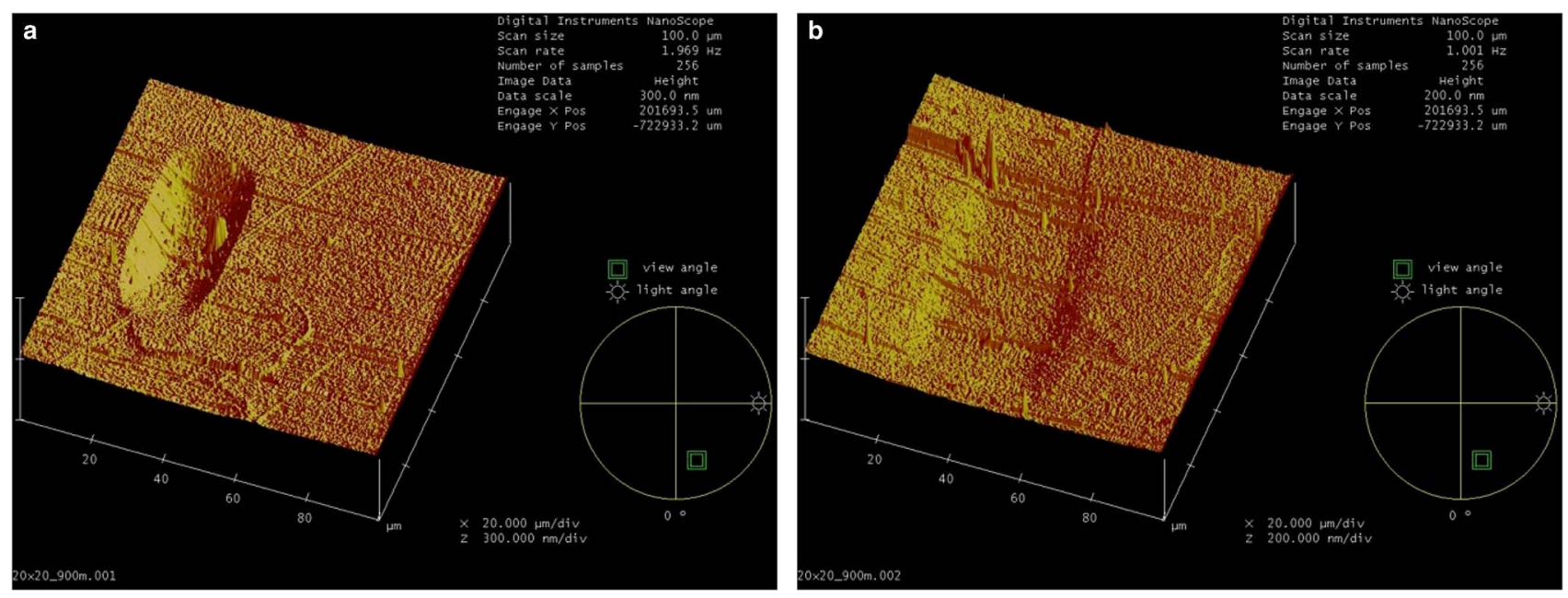

Figure 1. Si specimen topography after targeted $900 \mathrm{~V}$ Ar ion milling. a: $20 \times 20 \mu \mathrm{m}^{2}$ area. b: $40 \times 40 \mu \mathrm{m}^{2}$ area. Milling rates were 7.7 and $2.0 \mathrm{~nm} / \mathrm{min}$, respectively.
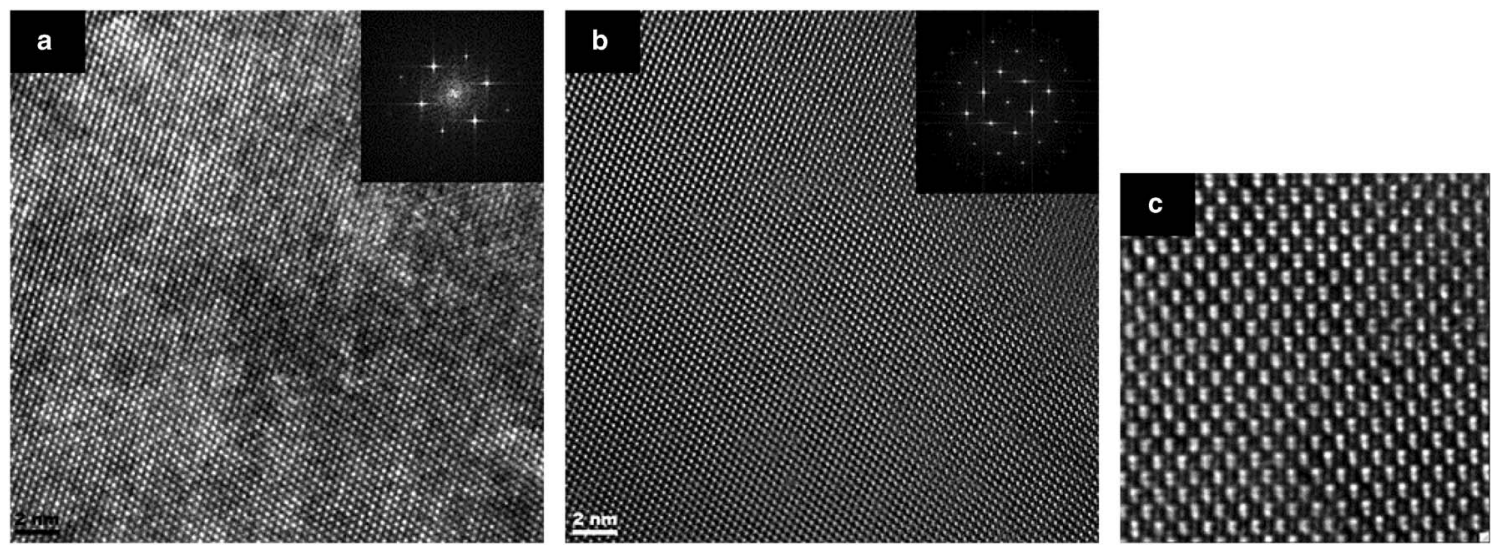

Figure 2. Si specimen before (a) and after (b) milling using a $200 \mathrm{eV}$ Ar ion beam. A final specimen thickness of $\sim 7 \mathrm{~nm}$ after milling resulted to sub-Ångström lattice spacing evident as shown in image (b) and fast Fourier transform (inset of b). c: Shows the $1.35 \AA$ dumbbell spacing of Si. Images (b) and (c) were acquired using the TEAM 0.5 at an operating voltage of $80 \mathrm{keV}$.

to measure these rates on $\mathrm{Si}(100)$ specimens and to show the shape of the milled areas, as presented in Figure 1. Depending upon the milling area chosen, milling rates for the $900 \mathrm{eV}$ Ar ion beam vary from 7.7 to $2.0 \mathrm{~nm} / \mathrm{min}$ for raster box sizes of $20 \times 20 \mu \mathrm{m}^{2}$ and $40 \times 40 \mu \mathrm{m}^{2}$, respectively.

\section{Silicon}

Silicon is the most widely used semiconducting material from which integrated circuits are created. Virtually all commercially available microelectronic devices are Si-based. Pure silicon is not a conductor; therefore, "doping" pure silicon with very small amounts of elements such as boron or phosphorus give it semiconducting properties that are ideal for electronic devices. Because of the ubiquitous nature of silicon in the semiconductor industry, a silicon specimen was prepared for this research. Figure 2 demonstrates that by thinning with a $200 \mathrm{eV}$ Ar ion beam, high-quality TEM specimens can be achieved that allow for imaging with sub-Ångström resolution.

\section{MnAs on GaAs Substrate}

MnAs is a room temperature ferromagnetic pnictide that can be easily grown in epitaxy with semiconductor substrates of GaAs and Si (Das et al., 2003). Figure 3a shows a bright-field transmission electron microscopy (BF-TEM) image of the FIB-prepared specimen before low-energy Ar ion milling. Figure $3 \mathrm{~b}$ displays a HRTEM image of the same location. The dark-contrast variation in GaAs in Figure $3 b$ is due to the presence of a damaged layer on the surface. The specimen was Ar ion milled from both sides at 900 and $500 \mathrm{eV}$ energies and incident angles of 10 and $12^{\circ}$, respectively. The Ar ion milling was precisely targeted on the specimen using the NanoMill system's SED; the SED image is shown in Figure 3c. Low-energy ion milling successfully removed most of the specimen damage, as shown in Figure 3d.

The high-energy sputtering process with Ga ions not only damages the surface of the specimen, but also makes it rough. 

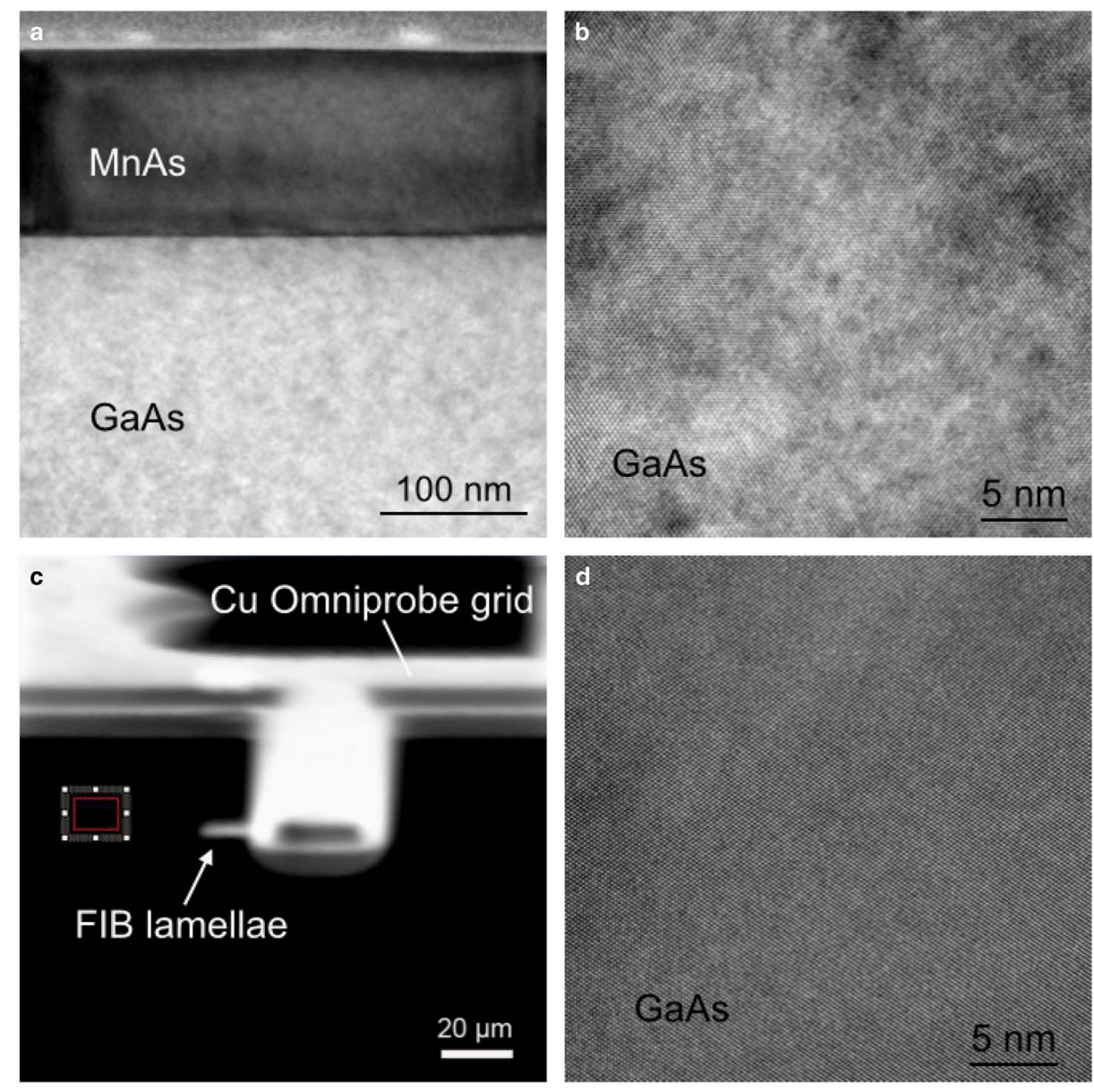

Figure 3. a: Bright-field transmission electron microscopy image of MnAs on GaAs after focused ion beam (FIB) preparation finished at $5 \mathrm{keV}$. b: High-resolution transmission electron microscopy (HRTEM) image of GaAs showing the patchy contrast due to surface amorphization and roughness. c: Image of the FIB lamella and Omniprobe grid in the NanoMill system. Ion beam energy is $900 \mathrm{eV}$, tilt $15^{\circ}$. d: HRTEM image of GaAs after ion milling at $500 \mathrm{eV}$ at $12^{\circ}$ for $30 \mathrm{~min}$.
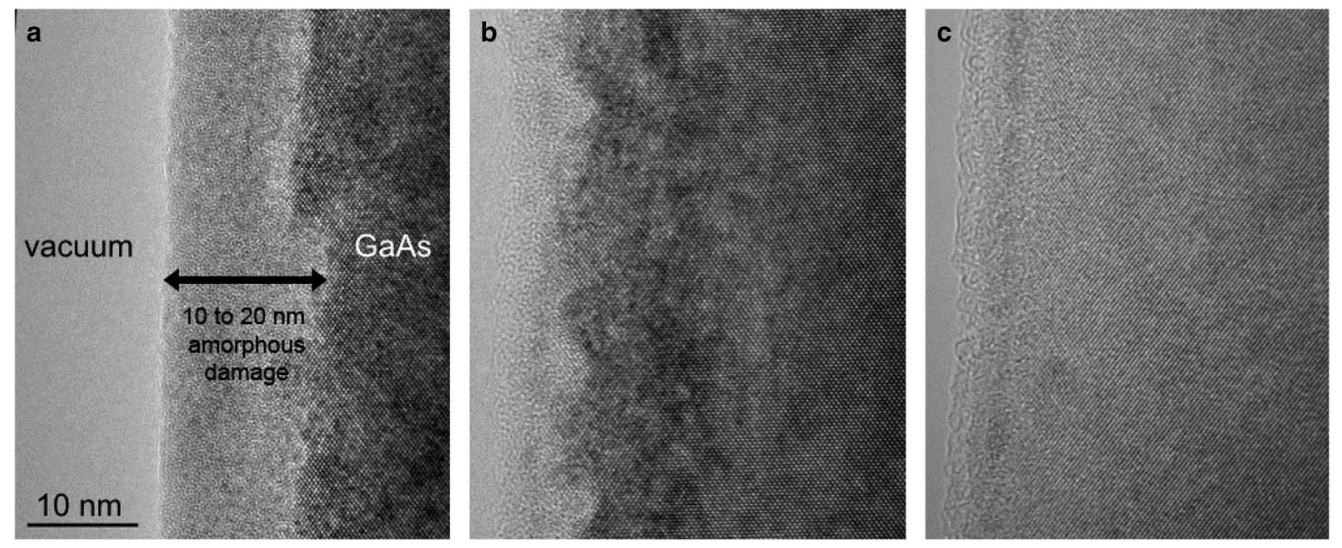

Figure 4. High-resolution transmission electron microscopy images of GaAs edge after (a) focused ion beam preparation at $5 \mathrm{keV}$, (b) Ar ion milling at $900 \mathrm{eV}$ for $10 \mathrm{~min}$ at $10^{\circ}$, and (c) Ar ion milling at $500 \mathrm{eV}$ for $20 \mathrm{~min}$ at $10^{\circ}$.

Figure 4 shows the improvement in specimen condition as it moves through the low-energy ion milling process. The $5 \mathrm{keV}$ energy $\mathrm{Ga}$ ion beam produced a 10 - to 20 -nm thick amorphous layer on GaAs and an inhomogeneous surface with $\sim 5 \mathrm{~nm}$ roughness, as shown in Figure $4 \mathrm{a}$, where the roughness can be estimated from the jagged edge of the 
crystalline part of the specimen. Ar ion milling at $900 \mathrm{eV}$ for 10 min removed most of the amorphous layer from the surface, but the milling process was not sufficiently long to remove all the FIB damage and the roughness remained relatively the same. Additional milling at $500 \mathrm{eV}$ removed almost all the amorphous GaAs from the surface and roughness was reduced, as well. The removal of the surface damage part of the specimen results in a reduced thickness, which may improve the image contrast of the BF-TEM image.

\section{Co: $\mathrm{ZnO}$ on $\mathrm{Al}_{2} \mathrm{O}_{3}$ Substrate}

Oxides are popular as substrates in thin-film growth technologies to generate various epitaxial layers. Oxide materials, in interaction with high-energy ions, typically form a lesser amount of an amorphous layer on the surface, but are more sensitive to selective sputtering, which may result in a high surface roughness. Figure 5 shows the images of Co-doped $\mathrm{ZnO}$ thin film (Kovács et al., 2013) deposited on $\mathrm{Al}_{2} \mathrm{O}_{3}$ (sapphire) as an example of substantial surface modification. The slightly defocused BF-TEM image of the substrate, Figure 5b, shows the nanometer-sized "pits" in sapphire after
FIB preparation at $5 \mathrm{keV}$, which are eliminated by the sequential milling of the specimen at 900 and $500 \mathrm{eV}$ with a concentrated Ar ion beam, as shown in Figures $5 c$ and $5 d$.

The vertical contrast lines visible in Figures $5 \mathrm{a}$, and $5 \mathrm{~b}$ are due to the so-called "curtaining effect" (Giannuzzi \& Stevie, 1999), which forms due to sputtering rate differences in the specimen. The main source of curtaining in this specimen was the relatively rough polycrystalline structure of the ion beam-deposited $\mathrm{Pt} / \mathrm{C}$ protective layer. The "curtaining" effect can be greatly reduced by depositing a homogenous protective layer on the top of the samples, for example, pure carbon or tungsten, in the FIB. In addition, the NanoMill system ion milling process minimizes the curtaining effect, as shown in Figures $5 c$ and $5 d$.

\section{Examples for Quantitative TEM (aberration corrected)}

In addition to the possibility of reducing the surface damage layer, the adjustment of the layer thickness is equally important for quantitative studies of material properties using aberration-corrected TEM, which often requires fixed
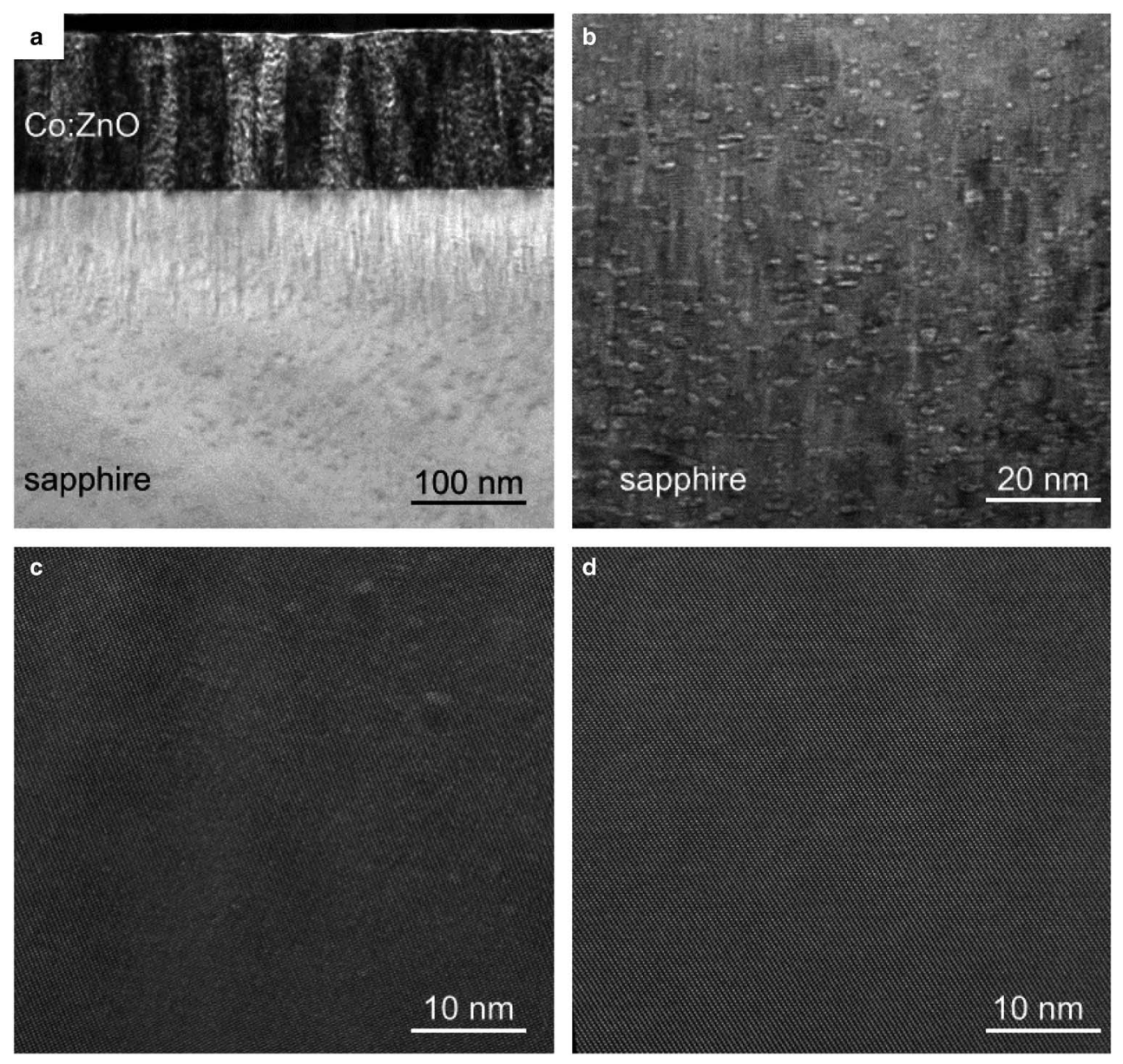

Figure 5. a: Bright-field transmission electron microscopy image of Co-doped $\mathrm{ZnO}$ thin film deposited on sapphire substrate after the focused ion beam preparation finished at $5 \mathrm{keV}$. The specimen thickness is $\sim 100 \mathrm{~nm}$. b: Magnified image of the sapphire substrate showing round shape contrast of hollow regions and line contrast of a curtaining effect. c: High-resolution transmission electron microscopy (HRTEM) image of sapphire after milling at $900 \mathrm{eV}$ for $5 \mathrm{~min}$ with Ar ions. d: HRTEM image of sapphire after milling at $500 \mathrm{eV}$ for an additional $15 \mathrm{~min}$ with Ar ions. 

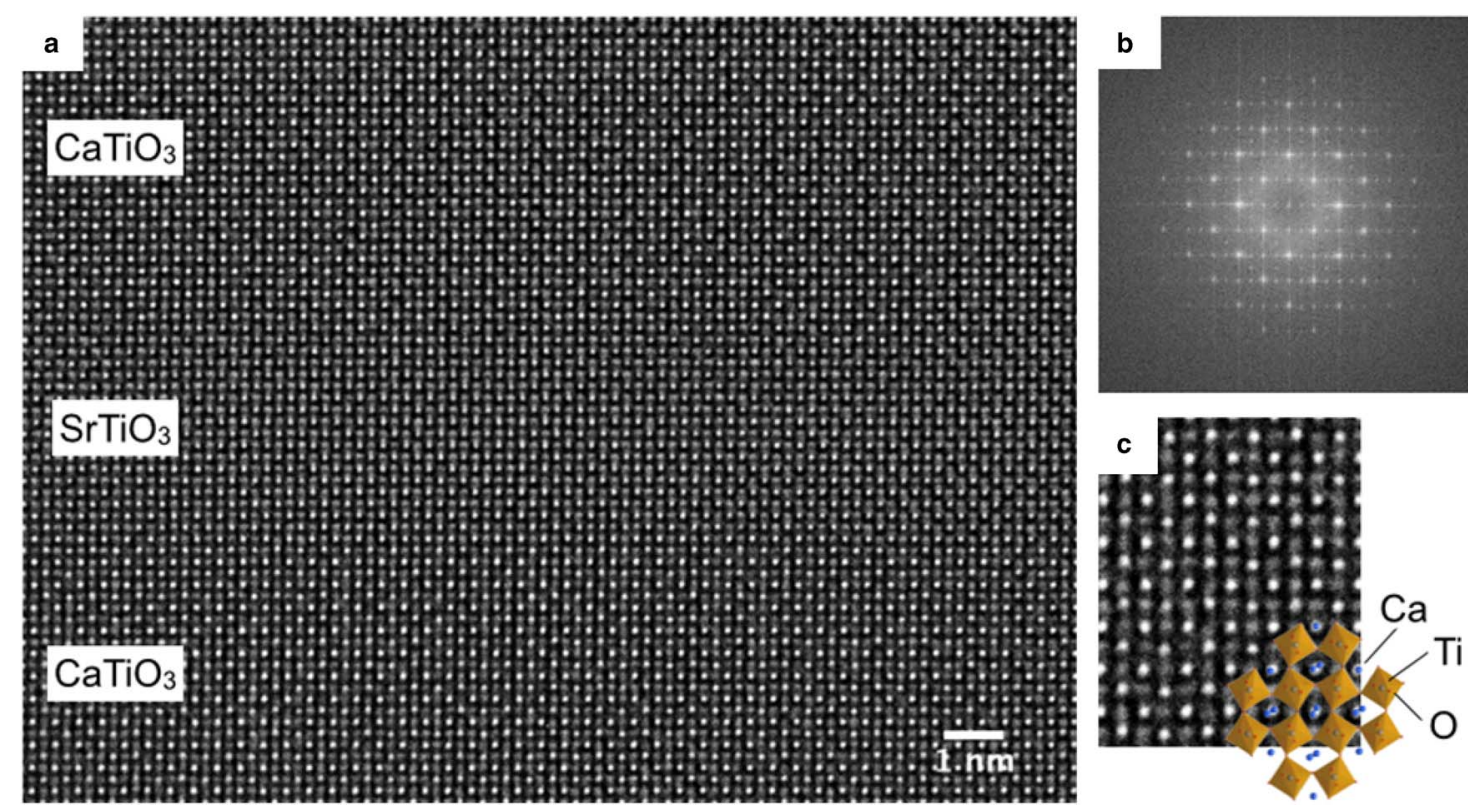

Figure 6. a: Unprocessed aberration-corrected high-resolution transmission electron microscopy image of $\mathrm{CaTiO}_{3}$ and $\mathrm{SrTiO}_{3}$ multilayer and its Fourier transform shown in (b). c: Part of the $\mathrm{CaTiO}_{3}$ layer displayed in larger magnification together with a schematic of the atomic structure. Clearly, oxygen columns are resolved.

and/or very thin specimen thickness-in some cases, $<10 \mathrm{~nm}$ (Jia et al., 2010). Specimen thickness is also an important factor in studies of beam-sensitive materials because they require low-acceleration voltages $(<100 \mathrm{keV})$ in the TEM.

Figure 6 shows a HRTEM image of a $\mathrm{CaTiO}_{3} / \mathrm{SrTiO}_{3}$ multilayer specimen recorded at negative $C_{s}$ conditions (Jia et al., 2010) using the spherical and chromatic aberrationcorrected microscope operated at $80 \mathrm{keV}$. The FIB lamellae preparation was done in accordance with the steps shown in Table 1, whereas the final milling was done at $500 \mathrm{eV}$ using Ar ions. Despite the low acceleration voltage, the atomic columns of $\mathrm{Sr}, \mathrm{Ti}$, and $\mathrm{Ca}$ are clearly resolved and relatively equal in brightness. In between these positions, weaker contrast produced by the oxygen columns are observed and shown in Figure 6c.

Although the HRTEM image represents the pinnacle of quantitative TEM imaging due to specimen and instrumentation; of equal importance is the more commonly applied technique of quantitative HAADF-STEM imaging (LeBeau et al., 2008; LeBeau et al., 2009; LeBeau et al., 2010a, $2010 b$ ). This technique requires linearization of the HAADF detector, which has shown that column intensity scales with atomic number $(Z) \sim Z^{1.6-1.9}$ (Hartel et al., 1996; Rafferty et al., 2001) through incoherent signal collection. For clean specimens $<10 \mathrm{~nm}$ in thickness, it is possible to chemically identify atomic columns determined by the corresponding HAADF intensity. A specimen that permits quantitative HAADF-STEM imaging and the corresponding column identification is shown in Figures $7 \mathrm{a}$ and $7 \mathrm{~b}$, respectively (Hauser et al., 2011). The HAADF image was acquired using a third-order aberration- and probecorrected Titan STEM equipped with an annular dark-field detector (E.A. Fischione Instruments, Inc.). The STEM was operated at $300 \mathrm{keV}$ and is presented with no image filtering to highlight the uniform imaging conditions possible following low-energy Ar ion milling, which reduces residual surface artifacts.

\section{Examples of Structural Metallurgical Alloys}

A common technique for the analysis of metallurgical specimens is the preparation of $3 \mathrm{~mm}$ discs by mechanical dimpling and broad-beam ion milling. The final surface quality of $3 \mathrm{~mm}$ discs and the corresponding analytical electron microscopy results can be improved with low-energy Ar ion milling. This approach is also useful for specimens that acquire surface oxidation during storage.

Results obtained for a Ni-based superalloy specimen that was dimpled mechanically and broad-beam milled (5 keV with Ar ions) are shown in Figure 8a. The progressive improvement in HRTEM image clarity and the reduction of surface mottling related to the smoothing of topography as the incident Ar ion beam energy is reduced from 5 to $500 \mathrm{eV}$ is observed in Figures $8 \mathrm{~b}$ and $8 \mathrm{c}$.

FIB preparation with a $\mathrm{Ga}$ ion beam for site specific, as well as crystallographically oriented, metallurgical specimens has become the preferred method for many researchers involved in characterizing deformation behavior along specific crystallographic planes. One of the most important aspects of structural alloy characterization when using the TEM is dislocation and defect analysis for correlation of the alloy's structure with mechanical behavior. Classically, this characterization was performed using diffraction contrast techniques and tilting to reveal the nature of the defects. 


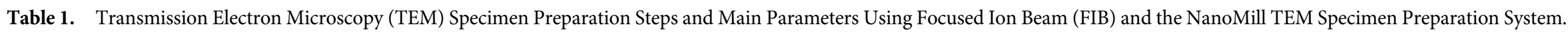

\begin{tabular}{|c|c|c|c|c|c|c|}
\hline & Specimen Preparation $^{c}$ & $\begin{array}{l}\text { Beam Energy } \\
(\mathrm{keV})\end{array}$ & $\begin{array}{l}\text { Beam } \\
\text { Current }\end{array}$ & $\begin{array}{l}\text { Incident } \\
\text { Angle }\end{array}$ & Size or Time & Remarks/Notes \\
\hline \multirow[t]{10}{*}{ FIB Milling ${ }^{\mathrm{a}}$} & $\mathrm{Pt} / \mathrm{C}$ protective layer deposition & 5 & $55 \mathrm{pA}$ & $0^{\circ}$ & & $\begin{array}{l}\text { - Electron beam used for deposition } \\
\text { - Use is material dependent }\end{array}$ \\
\hline & $\mathrm{C}$ protective layer deposition & 5 & $55 \mathrm{pA}$ & $0^{\circ}$ & & $\begin{array}{l}\text { - Electron beam used for deposition } \\
\text { - Use is material dependent }\end{array}$ \\
\hline & $\mathrm{Pt} / \mathrm{C}$ protective layer deposition & 30 & $0.28 \mathrm{nA}$ & $52^{\circ}$ & & Ga ion beam used \\
\hline & Trench cut & 30 & $21 \mathrm{nA}$ & $52^{\circ}$ & $\begin{array}{l}\text { Two trenches, each } \\
20 \times 8 \mu \mathrm{m}\end{array}$ & Lamellae thickness down to $\sim 3 \mu \mathrm{m}$ \\
\hline & J-undercut & 30 & $6.5 \mathrm{nA}$ & $7^{\circ}$ & & \\
\hline & $\begin{array}{l}\text { Lift out and weld the lamella to an Omniprobe } \\
\text { grid using a micromanipulator }\end{array}$ & & & & & $22.5 \times 1 \times 1.5 \mu \mathrm{m}$ Pt strip at $30 \mathrm{keV}, 48 \mathrm{pA}$ \\
\hline & Thinning & $\begin{array}{l}30 \\
30\end{array}$ & $\begin{array}{r}2.8 \mathrm{nA} \\
0.92 \mathrm{nA}\end{array}$ & $\begin{array}{l} \pm 2^{\circ} \\
+1.5^{\circ}\end{array}$ & & $\begin{array}{l}\text { Down to } \sim 1.5 \mu \mathrm{m}\left(\mathrm{CCS}^{\mathrm{e}}\right) \\
<1 \mu \mathrm{m}\left(\mathrm{CCS}^{\mathrm{e}}\right)\end{array}$ \\
\hline & & 30 & $0.46 \mathrm{nA}$ & $+1.5^{\circ}$ & & $<0.5 \mu \mathrm{m}\left(\mathrm{CCS}^{\mathrm{e}}\right)$ \\
\hline & & 30 & $93 \mathrm{pA}$ & $\pm 1.2^{\circ}$ & & $\sim 0.2 \mu \mathrm{m}$ (rectangular box) \\
\hline & Thinning & 5 & $47 \mathrm{pA}$ & $\pm 5^{\circ}$ & & $\sim 0.1 \mu \mathrm{m}$ (rectangular box $)$ \\
\hline \multirow[t]{4}{*}{$\begin{array}{l}\text { NanoMilling } \\
\text { process }^{b}\end{array}$} & Thinning $^{c}$ & 0.9 & $120 \mu \mathrm{A}^{\mathrm{d}}$ & $\pm 10^{\circ}$ & $\sim 30 \min$ & $\begin{array}{l}\text { - Current is measured using a Faraday cup } \\
\text { - Time is specimen dependent }\end{array}$ \\
\hline & Thinning $^{c}$ & 0.5 & $120 \mu \mathrm{A}^{\mathrm{d}}$ & $+12 /-10^{\circ}$ & $\sim 40 \mathrm{~min}$ & $\begin{array}{l}\text { - Current is measured using a Faraday cup } \\
\text { - Time is specimen dependent }\end{array}$ \\
\hline & Thinning $^{c}$ & $<0.5$ & $120 \mu \mathrm{A}^{\mathrm{d}}$ & $+14 /-10^{\circ}$ & $\sim 40 \mathrm{~min}$ & Beam-sensitive materials \\
\hline & Optional thinning at cryogenic temperature & $0.2-0.9$ & $120 \mu \mathrm{A}^{\mathrm{d}}$ & & & $\begin{array}{l}\text { - Beam-sensitive materials } \\
\text { - Materials containing polymers }\end{array}$ \\
\hline
\end{tabular}

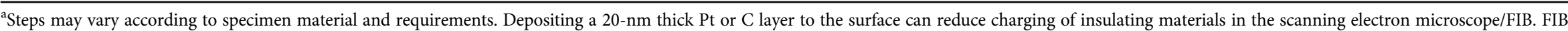

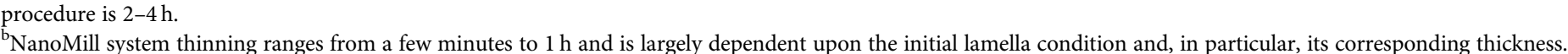

${ }^{\mathrm{c}}$ Milling time and incident angles depend on the material, damaged layer thickness, and required final thickness of the lamellae. Milling current is adjustable.

${ }^{\mathrm{d}}$ Emission current.

${ }^{\mathrm{e}} \mathrm{CCS}$, cleaning cross-section. 

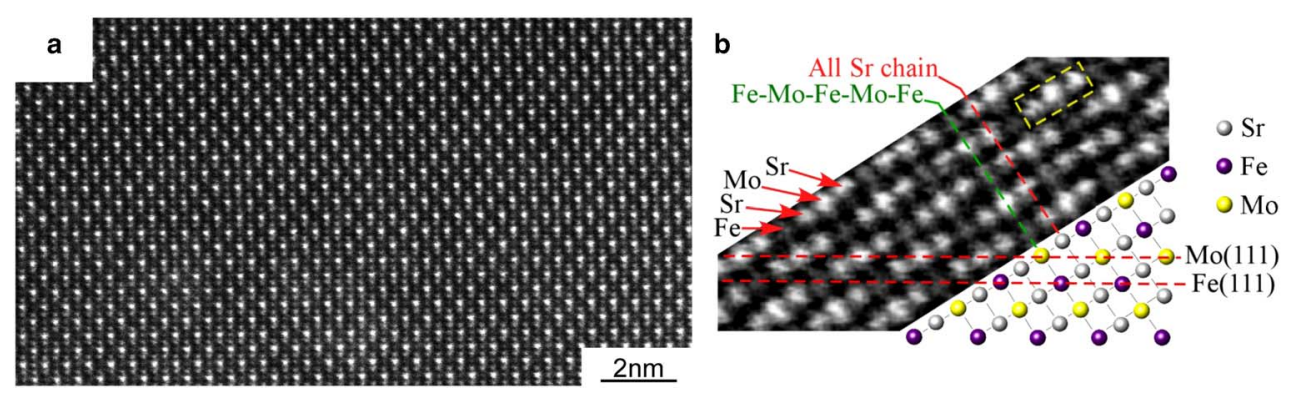

Figure 7. Unfiltered, aberration-corrected high-angle annular dark-field scanning transmission electron microscopy (HAADF-STEM) images of a $\mathrm{Sr}_{2} \mathrm{FeMoO}_{6}$ thin film grown on $\mathrm{SrTiO}_{3}$. a: Uniform, damage-minimized surface condition is shown. b: A schematic with the corresponding HAADF-STEM image showing the projection of the double perovskite ordering along the $\langle 110\rangle$ and the Mo-Fe ordering separated by a Sr chain (Hauser et al., 2011).
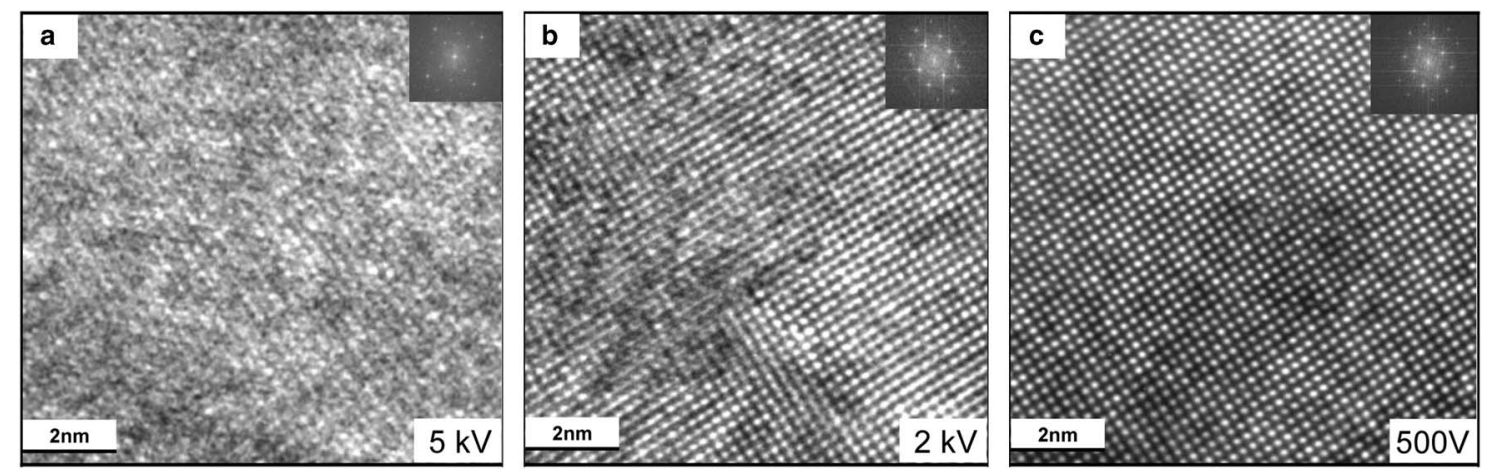

Figure 8. Improvement in high-resolution transmission electron microscopy images from a Ni-based superalloy specimen after successive applications of low-energy Ar ion milling are shown for the image quality following (a) broadbeam milling at $5 \mathrm{keV}$, (b) Ar ion milling at $2 \mathrm{keV}$ and (c) $500 \mathrm{eV}$. The improvement in image quality is characterized with the inset fast Fourier transform for each image.

While diffraction contrast has been and will continue to be a vital characterization method for structural alloys using the TEM, one disadvantage associated with the diffraction contrast technique for defect analysis involves the very basis of the technique, namely dynamical scattering. For example, when characterizing dislocation substructures, the presence of bend contours and surface damage can obscure large parts of an image, as shown in Figure 9a.

Mills and his colleagues have shown that significant advantages may be accrued by using STEM to reduce the extent of the dynamical contrast, while retaining the ability to characterize defects following the traditional "TEM rules" of diffraction contrast (Phillips et al., 2011). This STEM technique allows a rapid characterization of dislocation substructures and permits the analysis of much thicker specimens, which enables the collection of more robust data. Figure $9 \mathrm{~b}$ shows an increased magnification relative to Figure 9a, the oval indicates the same lath in both images. The dominant dark contrast, observed in Figure 9a, is indicative of the residual damage and amorphization that occurs during Ga ion milling. This diffraction contrast is prohibitive to analysis and must be removed for characterization of the salient microstructural features present in the alloy. The result of subsequent low-energy Ar ion milling of the surface at $500 \mathrm{eV}$ is shown in Figure $9 \mathrm{~b}$ and reveals the dislocation substructure desired for analysis.

Not unexpectedly, the residual surface modifications from Ga ion milling also manifest in STEM diffraction contrast techniques and can prohibit characterization, as shown in Figure 10a. Figures 10a and 10c are bright-field STEM images of a structural titanium alloy. The subsequent improvement in imaging quality with decreasing ion milling beam energy is evident in Figure 10c-clear separation of dislocations and no undesirable contrast modulations in the matrix.

Specimen preparation artifacts can be resolution limiting for analytical TEM, in part due to surface modification and/or damage of the lattice, which results in scattering of the electron beam in an undesirable fashion and obscures microstructural features. FIB preparation is increasingly ubiquitous; thus, to prepare high-quality TEM specimens for qualitative and quantitative methods as shown above, low-kilovolt $\mathrm{Ar}$ ion milling is critical. The modified specimen surface layer may exhibit an altered chemical composition at interfaces of interest as a result of Ga FIB preparation. Our results show that uniform and damage-free specimens can be produced using the NanoMill system; a small spot from sufficiently low ion energy (as low as $50 \mathrm{eV}$ ) produces enough current $(\sim 100-150 \mathrm{pA})$ to allow for reasonable milling rates $(\sim 1-8 \mathrm{~nm} / \mathrm{min})$. 

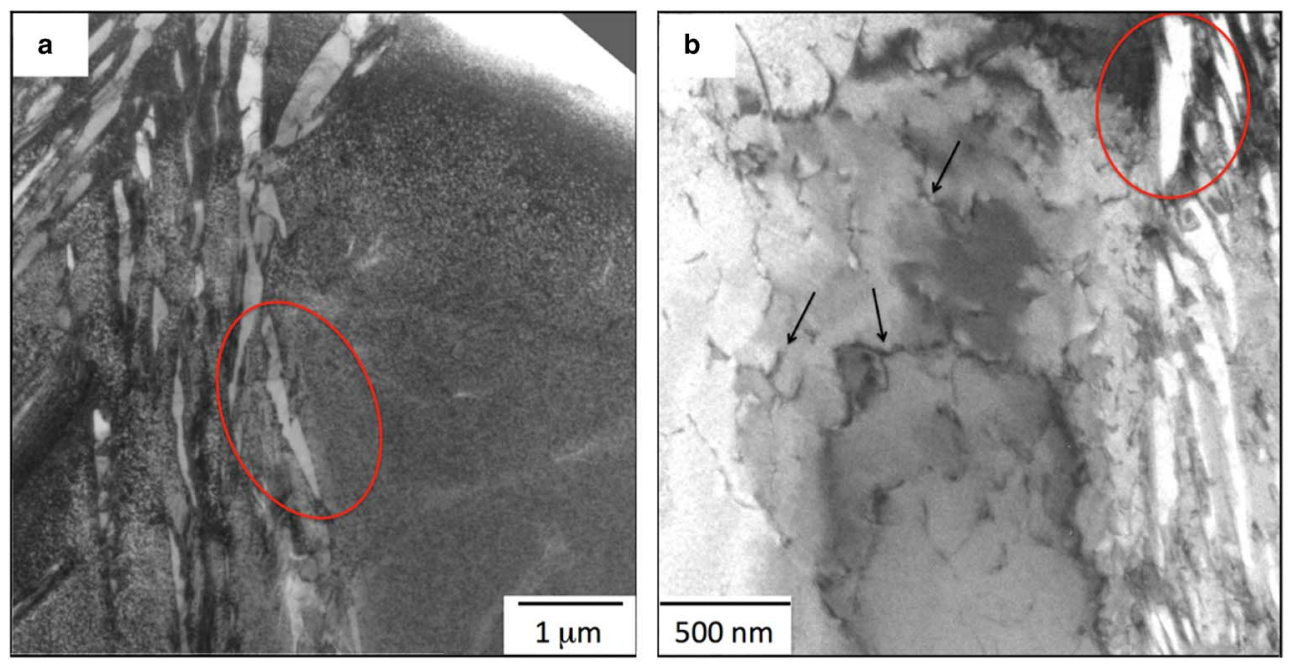

Figure 9. Conventional diffraction contrast images of a structural titanium alloy taken at different magnifications and areas showing the lath feature labeled in (a) and (b). The arrows in (b) indicate the dark linear features as dislocations. Note the marked reduction in "black spot" contrast when compared with (a), which permits clear observation of dislocations in (b) after NanoMilling.
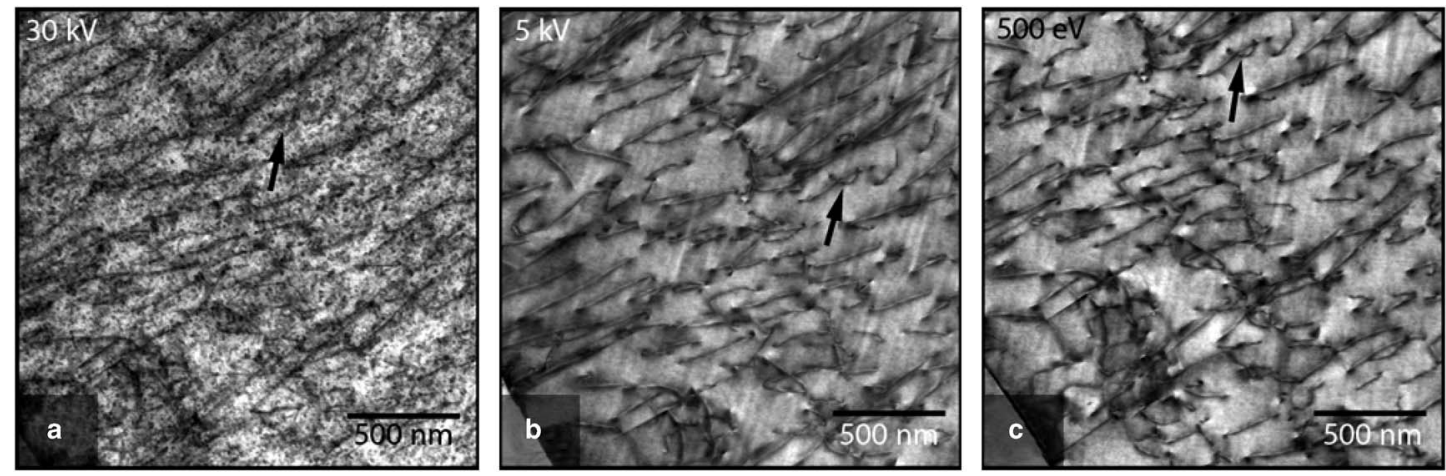

Figure 10. Bright-field scanning transmission electron microscopy images of a structural titanium alloy characterizing the dislocation structure. The arrows indicate the same dislocation in (a), (b), and (c). The black spots in (a) are indicative of diffraction contrast from $30 \mathrm{keV} \mathrm{Ga}$ ion surface modification and obscure the desired dislocation information. Although the image improved after $5 \mathrm{keV}$ ion milling, the separation between dislocations is not as evident as observed in (c). The intensity variation between matrix and dislocation is maximized after $500 \mathrm{eV}$ milling, (c).

\section{Conclusions}

State-of-the-art transmission electron microscopy often requires thin $(<10 \mathrm{~nm})$ specimens where possible amorphous surface layers are, at most, $0.5 \mathrm{~nm}$. To match these criteria, FIB-prepared lamellae, for example, require post treatment by $\mathrm{Ar}$ ion milling to eliminate spurious results. In this work, an $\sim 1 \mu \mathrm{m}$ FWHM diameter, lowenergy, concentrated, Ar ion beam successfully reduced the thickness of ion-damaged surface layers and prevented redeposition of sputtered material. Experiments revealed significant improvement in image quality for various materials of technological and scientific interest, which enabled the collection of accurate, quantitative metrics using a variety of analytical electron microscopy techniques, ranging from diffraction contrast to quantitative STEM imaging.

\section{ACKNOWLEDGMENTS}

The authors thank D. Meertens (Forschungszentrum Jülich, PGI-5) for the preparation of FIB lamellas. The MnAs and $\mathrm{ZnO}$ specimens were kindly provided by A. Ney (J. Kepler University, Linz, Austria). Appreciation is also extended to Joachim Mayer and Christian Kisielowski for their efforts in preparing and conducting the microscopy for the $\mathrm{Si}$ specimen.

\section{REFERENCES}

ANDERSON, R. \& KLePeIS, S.J. (2005). Practical aspects of FIB TEM specimen preparation. In Introduction to Focused Ion Beams: Instrumentation, Theory, Techniques, and Practice, Giannuzzi, L.A. \& Stevie, F.A. (Eds.), pp. 173-200. New York, NY: Springer. 
BAHNCK, D. \& Hull, R. (1990). Experimental measurement of transmission electron microscope specimen temperature during ion milling. MRS Online Proc Libr 199, 253.

BARBER, D.J. (1993). Radiation damage in ion-milled specimens: Characteristics, effects and methods of damage limitation. Ultramicroscopy 52(1), 101-125.

Barna, Á. (1991). Topographic kinetics and practice of low angle ion beam thinning. MRS Online Proc Libr 254, 3-22.

Barna, Á., Pécz, B. \& Menyhard, M. (1998). Amorphisation and surface morphology development at low-energy ion milling. Ultramicroscopy 70(3), 161-171.

BARna, Á., PÉCZ, B. \& MENYhARD, M. (1999). TEM sample preparation by ion milling/amorphization. Micron 30(3), 267-276.

Barnard, A.W., Hyun, J.K., Grazul, J.L. \& Muller, D.A. (2006). Surface roughness instabilities in low-angle ion milling. Microsc Microanal 12(S02), 1318-1319.

Basile, D.P., Boylan, R., Baker, B., Hayes, K. \& Soza, D. (1991). Fibxtem-Focussed ion beam milling for TEM sample preparation. MRS Online Proc Libr 254, 23-41.

Batson, P.E., Dellby, N. \& KrivaneK, O.L. (2002). Sub-Ångström resolution using aberration corrected electron optics. Nature 418(6898), 617-620.

Cooper, D., Truche, R., Twitchett-Harrison, A., Dunin-Borkowski, R.E. \& Midgley, P. (2009). Quantitative off-axis electron holography of GaAs p-n junctions prepared by focused ion beam milling. J Microsc 233(1), 102-113.

Das, A.K., Pampuch, C., Ney, A., Hesjedal, T., Däweritz, L., Koch, R. \& Ploog, K.H. (2003). Ferromagnetism of MnAs studied by heteroepitaxial films on GaAs(001). Phys Rev Lett 91(8), 087203.

Genç, A., Ohio State University \& E.A. Fischione Instruments InC (2007). Post-FIB TEM sample preparation using a low energy argon beam. Microsc Microanal 13(S02), 1520-1521.

GiannuzZI, L.A. (2006). Reducing FIB damage using low energy ions. Microsc Microanal 12(S02), 1260-1261.

GiannuzZi, L.A. \& Stevie, F.A. (1999). A review of focused ion beam milling techniques for TEM specimen preparation. Micron 30(3), 197-204.

Hartel, P., Rose, H. \& Dinges, C. (1996). Conditions and reasons for incoherent imaging in STEM. Ultramicroscopy 63(2), 93-114.

Hauser, A.J., Williams, R.E., Ricciardo, R.A., Genç, A., Dixit, M., Lucy, J.M., Woodward, P.M., Fraser, H.L. \& YanG, F. (2011). Unlocking the potential of half-metallic Sr2FeMoO6 films through controlled stoichiometry and double-perovskite ordering. Phys Rev B 83(1), 014407.

Huh, Y., Hong, K. \& SHIN, K. (2013). Amorphization induced by focused ion beam milling in metallic and electronic materials. Microsc Microanal 19(S5), 33-37.

Jia, C.L., Houben, L., Thust, A. \& Barthel, J. (2010). On the benefit of the negative-spherical-aberration imaging technique for quantitative HRTEM. Ultramicroscopy 110(5), 500-505.

JiA, C.L., Mi, S.B., Barthel, J., WANG, D.W., Dunin-Borkowski, R.E., URBAN, K.W. \& Thust, A. (2014). Determination of the 3D shape of a nanoscale crystal with atomic resolution from a single image. Nat Mater 13(11), 1044-1049.

Kamino, T., Yaguchi, T., Hashimoto, T., Ohnishi, T. \& Umemura, K. (2005). A FIB micro-sampling technique and a site specific TEM specimen preparation method. In Introduction to Focused Ion Beams: Instrumentation, Theory, Techniques, and Practice, Giannuzzi, L.A. \& Stevie, F.A. (Eds.), pp. 229-245. New York, NY: Springer Science+Business Media, Inc.
Kato, N.I. (2004). Reducing focused ion beam damage to transmission electron microscopy samples. J Electron Microsc 53(5), 451-458.

Kirk, E.C., Williams, D.A. \& Ahmed, H. (1989). Cross-sectional transmission electron microscopy of precisely selected regions from semiconductor devices. Inst Phys Conf Ser 100, 501-506.

Kovács, A., Ney, A., Duchamp, M., Ney, V., Boothroyd, C.B., Galindo, P.L., Kaspar, T.C., Chambers, S.A. \& Dunin-Borkowski, R.E. (2013). Defects in paramagnetic Co-doped $\mathrm{ZnO}$ films studied by transmission electron microscopy. J Appl Phys 114, 243503.

LeBeau, J.M., D’Alfonso, A.J., Findlay, S.D., Stemmer, S. \& Allen, L.J. (2009). Quantitative comparisons of contrast in experimental and simulated bright-field scanning transmission electron microscopy images. Phys Rev B 80(17), 174106.

LeBeau, J.M., Findlay, S.D., Allen, L.J. \& Stemmer, S. (2008). Quantitative atomic resolution scanning transmission electron microscopy. Phys Rev Lett 100(20), 206101.

LeBeau, J.M., Findlay, S.D., Allen, L.J. \& Stemmer, S. (2010a). Position averaged convergent beam electron diffraction: Theory and applications. Ultramicroscopy 110(2), 118-125.

LeBeau, J.M., Findlay, S.D., Allen, L.J. \& Stemmer, S. (2010b). Standardless atom counting in scanning transmission electron microscopy. Nano Lett 10(11), 4405-4408.

LI, L. \& YANG, J.C. (2002). Oxide structures formed on silver single crystals due to hyperthermal atomic oxygen exposure. MRS Online Proc Libr 751, Z3.37.

Lotnyk, A., Poppitz, D., Ross, U., Gerlach, J., Frost, F., Bernütz, S., Thelander, E. \& Rauschenbach, B. (2015). Focused high- and low-energy ion milling for TEM specimen preparation. Microelectron Reliab 55(9-10), 2119-2125.

Mayer, J., Giannuzzi, L.A., Kamino, T. \& Michael, J. (2007). TEM sample preparation and FIB-induced damage. MRS Bull 32(5), 400-407.

McCaffrey, J.P., Phaneuf, M.W. \& Madsen, L.D. (2001). Surface damage formation during ion-beam thinning of samples for transmission electron microscopy. Ultramicroscopy 87, 97-104.

Mehrtens, T., Bley, S., Venkata Satyam, P. \& Rosenauer, A. (2012). Optimization of the preparation of GaN-based specimens with lowenergy ion milling for (S)TEM. Micron 43(8), 902-909.

Miyajima, N., Holzapfel, C., Asahara, Y., Dubrovinsky, L., Frost, D., Rubie, D., Drechsler, M., Niwa, K., Ichihara, M. \& Yagi, T. (2010). Combining FIB milling and conventional Argon ion milling techniques to prepare high-quality site-specific TEM samples for quantitative EELS analysis of oxygen in molten iron. J Microsc 238(3), 200-209.

Mkhoyan, K., Babinec, T., Maccagnano, S., Kirkland, E. \& Silcox, J. (2007). Separation of bulk and surface-losses in low-loss EELS measurements in STEM. Ultramicroscopy 107(4-5), 345-355.

MoberlyChan, W.J., Adams, D.P., Aziz, M.J., Hobler, G. \& Schenkel, T. (2007). Fundamentals of focused ion beam nanostructural processing: Below, at, and above the surface. MRS Bull 32(5), 424-432.

Phillips, P.J., Brandes, M.C., Mills, M.J. \& De Graef, M. (2011). Diffraction contrast STEM of dislocations: Imaging and simulations. Ultramicroscopy 111(9-10), 1483-1487.

Rafferty, B., Nellist, D. \& Pennycook, J. (2001). On the origin of transverse inchoherence in Z-contrast STEM. J Electron Microsc 50(3), 227-233.

Schaffer, M., Schaffer, B. \& Ramasse, Q. (2012). Sample preparation for atomic-resolution STEM at low voltages by FIB. Ultramicroscopy 114, 62-71. 
Scheu, C., Gao, M., Van Benthem, K., Tsukimoto, S., Schmidt, S., Sigle, W., Richter, G. \& Thomas, J. (2003). Advances in EELS spectroscopy by using new detector and new specimen preparation technologies. J Microsc 210(1), 16-24.

Unocic, K.A., Mills, M.J. \& Daehn, G.S. (2010). Effect of gallium focused ion beam milling on preparation of aluminium thin foils. J Microsc 240(3), 227-238.
Utke, I., Hoffmann, P. \& Melngailis, J. (2008). Gas-assisted focused electron beam and ion beam processing and fabrication. $J$ Vac Sci Technol B 26(4), 1197-1276.

VolKert, C.A. \& Minor, A.M. (2007). Focused ion beam microscopy and micromachining. MRS Bull 32(5), 389-399.

Young, R.J., KiRK, E.C., Williams, D.A. \& AhmEd, H. (1990). Fabrication of planar and cross-sectional TEM specimens using a focused ion beam. MRS Online Proc Libr 199, 205-216. 